\title{
Psychiatric emergency? What to consider before prescribing
}

\author{
Raj K. Kalapatapu, MD, PhD
}

Dr. Kalapatapu is Associate Professor of Psychiatry, University of California, San Francisco, School of Medicine; and Attending Psychiatrist, Psychiatric Emergency Services, Zuckerberg San Francisco General Hospital and Trauma Center, San Francisco, California.

\section{Disclosure}

The author reports no financial relationships with any companies whose products are mentioned in this article, or with manufacturers of competing products.

doi: 10.12788/cp.0037

\section{f}

Discuss this article at www.facebook.com/ MDedgePsychiatry
$P$ sychiatric emergencies-such as a patient who is agitated, selfdestructive, or suicidal-may arise in a variety of settings, including emergency departments and inpatient units. ${ }^{1}$ Before emergently prescribing psychotropic medications to address acute psychiatric symptoms, there are numerous factors a clinician needs to consider. ${ }^{1-3}$ Asking the following questions may help you quickly obtain important clinical information to determine which medication to use during a psychiatric emergency:

Age. Is the patient a child, adolescent, adult, or older adult?

Allergies. Does the patient have any medication allergies or sensitivities?

Behaviors. What are the imminent dangerous behaviors that warrant emergent medication use?

Collateral information. If the patient was brought by police or family, how was he/she behaving in the community or at home? If brought from a correctional facility or other institution, how did he/she behave in that setting?

\section{Concurrent diagnoses/interventions. Does} the patient have a psychiatric or medical diagnosis? Is the patient receiving any pharmacologic or nonpharmacologic treatments?

First visit. Is this the patient's first visit to your facility? Or has the patient been to the facility previously and/or repeatedly? Has the patient ever been prescribed psychotropic medications? If the patient has received emergent medications before, which medications were used, and were they helpful?

Legal status. Is the patient voluntary for treatment or involuntary for treatment? If voluntary, is involuntary treatment needed?

Street. Was this patient evaluated in a medical setting before presenting to your facility? Or did this patient arrive directly from the community/street?

Substance use. Has the patient been using any licit and/or illicit substances?

In my experience with psychiatric emergencies, asking these questions has helped guide my decision-making during these situations. They have helped me to determine the appropriate medication, route of administration, dose, and monitoring requirements. Although other factors can impact clinicians' decision-making in these situations, I have found these questions to be a good starting point.

\footnotetext{
References

1. Mavrogiorgou P, Brüne M, Juckel G. The management of psychiatric emergencies. Dtsch Arztebl Int. 2011;108(13) 222-230.

2. Glick RL, Berlin JS, Fishkind AB, et al (eds). Emergency psychiatry: principles and practice. 2nd ed. Philadelphia PA: Wolter Kluwer; 2020.

3. Garriga M, Pacchiarotti I, Kasper S, et al. Assessment and management of agitation in psychiatry: expert consensus. World J Biol Psychiatry. 2016;17(2):86-128.
} 